\title{
DESIGN OF THE CEBAF ENERGY UPGRADE CRYOMODULE COLD MASS*
}

\author{
J. Hogan ${ }^{\dagger}$, I. E. Campisi, J. Delayen, E. Feldl, R. Getz, A. Guerra, J. Henry, J. Mammosser, \\ L. Phillips, J. Preble, W. J. Schneider, K. Smith, J. Takacs, L. Turlington, M. A. Wiseman \\ Thomas Jefferson National Accelerator Facility, Newport News, Virginia 23606, USA
}

\begin{abstract}
The Jefferson Laboratory's CEBAF Energy Upgrade to $12 \mathrm{GeV}$ will be accomplished by employing new cryomodules with an increase in effective length of 40 percent while maintaining the same nominal cryomodule slot length within the linac. This is being achieved by changing from an eight five-cell cavity structure to an eight seven-cell cavity structure and removing all bellows between the cavities along the beamline. This approach has presented significant challenges with respect to the design as it pertains to the cooldown, support, alignment and tuning criteria. The solutions to these design challenges will be presented with an emphasis on cavity operational criteria along with the integrated support components to include higher order mode couplers, fundamental power couplers and tuning accommodations for a one-hertz resolution.
\end{abstract}

\section{INTRODUCTION}

The continuous electron beam accelerator at Jefferson Lab was originally designed to operate at $4 \mathrm{GeV}$. However this design has been able to produce and deliver beam energy as high as $6 \mathrm{GeV}$. There are currently ten empty cryomodule slots in the recirculating accelerator. Looking to the future needs of the physics community, Jefferson Lab decided to develop the next generation continuous electron beam accelerator capable of operating at energy levels in excess of $12 \mathrm{GeV}$ [1]. This limit was in part chosen due to the limitations of the existing magnets in the arcs to control the beam.

A new cryomodule design was needed for these higher energies that was capable of providing higher average gradients along with an increase in the active length. An eight seven-cell cavity structure per cryomodule was determined to be the optimum configuration to meet these requirements while accommodating the existing accelerator cryomodule slot length.

\section{CHALLENGES}

The cavity string consisting of eight seven-cell cavities is assembled in a clean room and hermetically sealed with two UHV gate valves at each end (Figure 1). Due to the addition of 16 cells to the beamline, which resulted in a forty percent increased in active length per cryomodule, the bellows between each cavity were eliminated. All

\footnotetext{
*Work supported by the U.S. Department of Energy under contract DE-AC05-84-ER40150

thogan@jlab.org
}

beamline movement including cooldown and tuning must be accommodated by the beamline bellows in the cold to warm transitions at each end of the cryomodule. To limit this beamline transitional motion so as not to over-load one bellows more than the other, the cold mass is anchored to the spaceframe (Figure 2) at the axial midpoint of the cryomodule. Table 1 shows the most extreme movement required by each bellows. The elimination of the beamline bellows also affects how the cold mass is supported in order to maintain the onemillimeter diameter cavity alignment tolerance required for beam stability and performance.

Table 1: Bellows Expansion Requirements

\begin{tabular}{|l|l|}
\hline Component (Four Cavities) & Displacement \\
\hline Cavity Cooldown & $6 \mathrm{~mm}$ \\
\hline Tuner Range & $3 \mathrm{~mm}$ \\
\hline Fabrication Tolerance & $4 \mathrm{~mm}$ \\
\hline Total Travel & $13 \mathrm{~mm}$ \\
\hline
\end{tabular}

Each cavity is housed in an individual helium vessel with all vessels in a cryomodule cooled down in parallel. Heat treating each cavity eliminates the risk of Q disease associated with a slow cooldown. Due to the narrow bandwidth of the cavities, the tuner is required to provide a $400 \mathrm{kHz}$ range with a one-hertz resolution. With a tuning coefficient of $250 \mathrm{kHz} / \mathrm{mm}$ this is equivalent to a mechanical resolution of 4 nanometers.

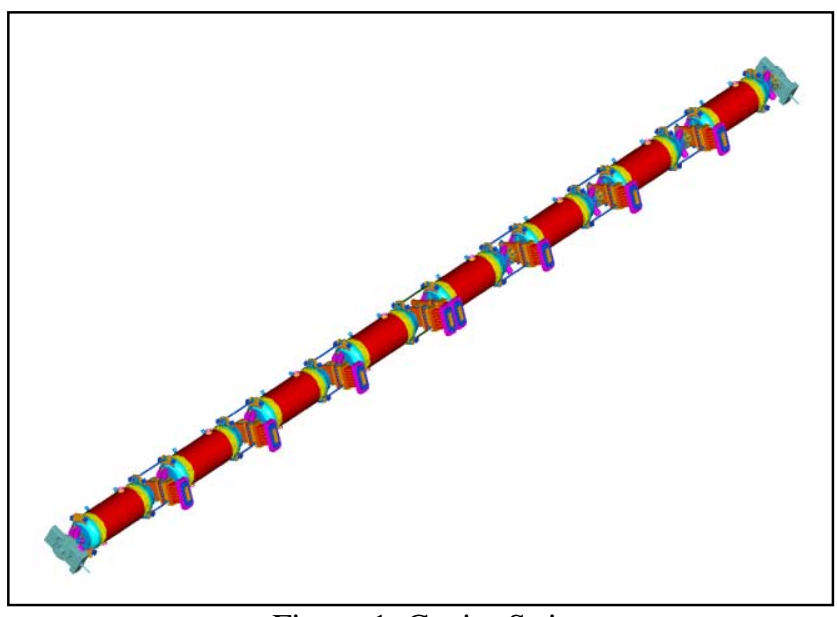

Figure 1. Cavity String

Due to the operational movement of the cold mass relative to the vacuum vessel, all connection and 
penetration designs must account for both the one time cooldown and the continuous tuning of the cavities.

\section{DESIGN DECISIONS}

The cavity string is mechanically anchored in the axial center of the cryomodule to control and limit the beamline movement (Figure 2). With no bellows between the cavities, the tuning operation must be carried through the rigid beamline without adversely affecting the frequency of the adjacent cavity. To eliminate any detrimental effects on the RF power coupling to the cavity each section of beamline between the cavities is connected with two titanium rods to carry these axial loads (Figure 3).

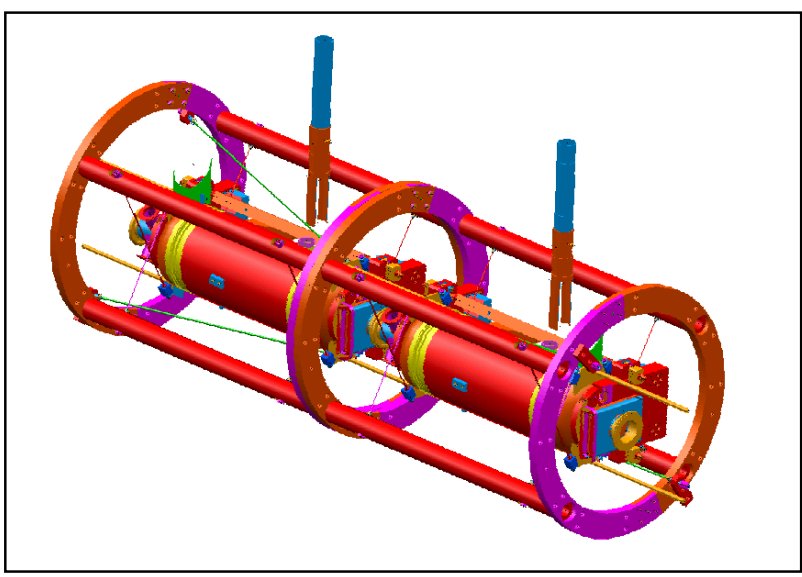

Figure 2. Center Anchor to Spaceframe

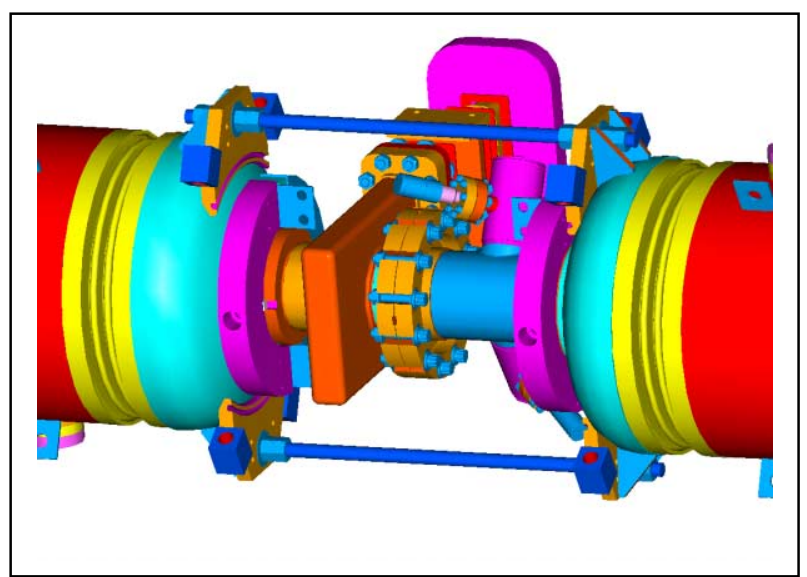

Figure 3. Cavity Beamline

The helium vessels are made of titanium to match the thermal contraction of the cavity during cooldown as well as decrease total weight. The titanium vessels are smaller than the original CEBAF helium vessels to limit the helium inventory. Additional helium inventory is contained in a five-inch header for control purposes and off normal conditions. Each helium vessel has two integrated titanium hydro-formed bellows to facilitate tuning and transient thermal contraction during cooldown (Figure 4).

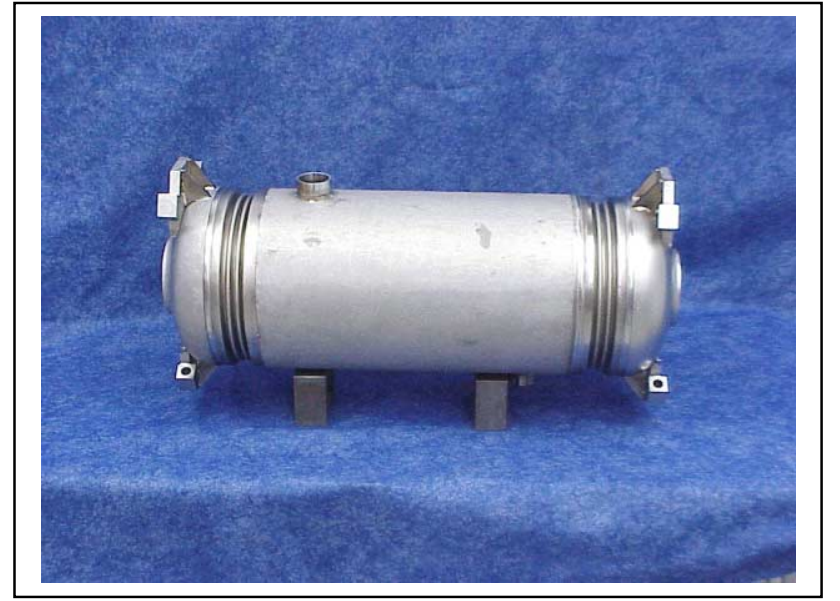

Figure 4. Titanium Helium Vessel

Each helium vessel in the cavity string is supported via eight high strength austenitic (Nitronic ${ }^{\circledR} 50$ ), $0.5 \mathrm{~cm}$ diameter rods, arranged in the same double- $X$ pattern used on the CEBAF cryomodule design. These supports will be offset during installation to account for the cooldown movement and will accommodate the operational tuning through a swivel effect. The support rods are connected to the helium vessel adjacent to the end cells of each cavity to assure accurate beamline reference. The rods are finitely adjustable to achieve a cavity string alignment tolerance of $\pm 0.5 \mathrm{~mm}$ in the transverse directions. The support rods as well as all components transitioning to room temperature are thermally intercepted to the $50 \mathrm{~K}$ insulation shield.

The tuner uses no cold bearing components; with the motor and drive are outside the vacuum vessel. A mechanical scissor jack design [2] is operated with the relative movement of a rod inside of a tube with each attached to the bottom and top of the mechanism respectively (Figures $5 \& 6$ ).

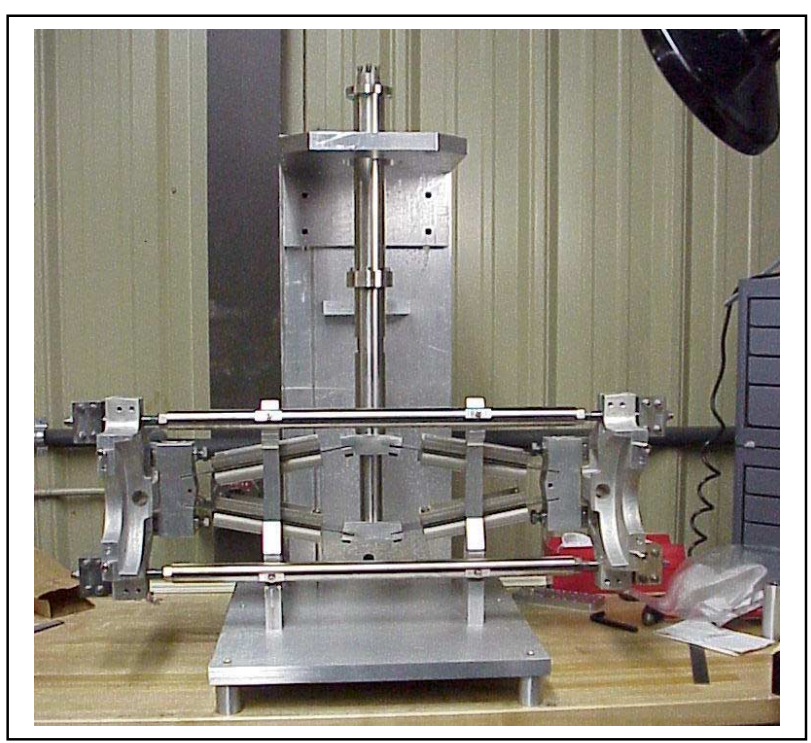

Figure 5. CEBAF Energy Upgrade Tuner 
This tuning system is designed to use a Piezo electric actuator for fine-tuning, however the mechanical design alone has proved through testing to be capable of achieving the required one-hertz resolution [2].

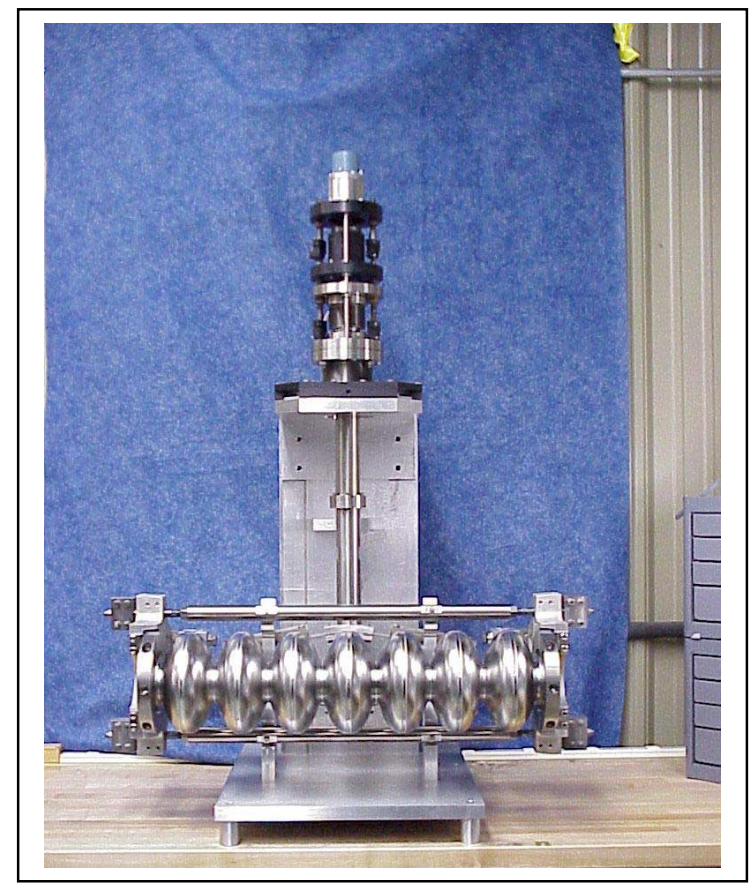

Figure 6. Bench Top Tuner \& Cavity

The waveguides have been designed with a double bellows and thermal intercept to the shield circuit. Taps have been installed on the $2 \mathrm{~K}$ circuits to incorporate active cooling of the waveguide and HOM if necessary in the future when requests for increased power are anticipated.

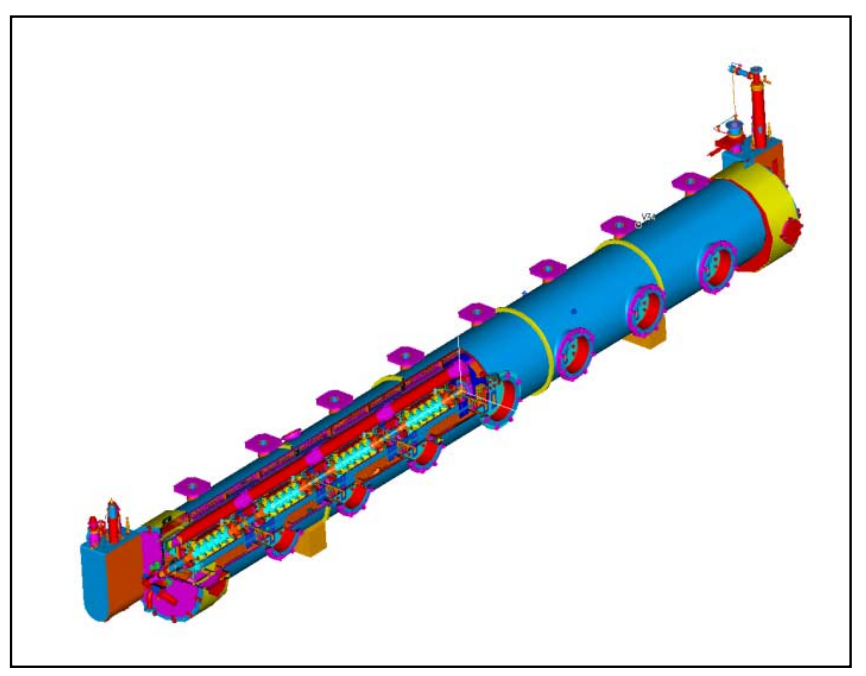

Figure 7. CEBAF Energy Upgrade Cryomodule

\section{CURRENT STATUS}

The first cavity string will be assembled in the JLAB clean room in the third quarter of 2001 with the first cryomodule (Figure 7) testing to be done in 2002. All major component contracts are awarded with partial deliveries already in house. All cavity fabrication and processing is performed at Jefferson Lab. Vertical testing of the first cavities is in progress.

The cavity cell shape is undergoing analysis to investigate higher gradients. Testing on the higher order mode couplers design is ongoing for both the free electron laser requirements as well as the CEBAF requirements.

The cryomodule-testing cave is undergoing a complete renovation including data acquisition hardware, klystron power and magnetic shielding. In addition the test cave must accommodate five cryomodule designs including the original CEBAF design, the JLAB energy upgrade design as well as the medium and high beta SNS cryomodules and a quarter cryomodule.

\section{ACKNOWLEDGEMENTS}

- The JLAB mechanical engineering department for their dedication and teamwork.

- The JLAB Superconducting Radio Frequency R\&D group for their world-class knowledge and aptitude.

- The JLAB administrative staff for their skill, professionalism and patience.

\section{REFERENCES}

[1] J. Preble, et al., "Cryomodule Development for CEBAF Upgrade," PAC'99.

[2] G. Davis, et al., "Development and Testing of a Prototype Tuner for the CEBAF Upgrade Cryomodule," PAC'2001. 